\title{
ON A CONSTRAINED MINIMIZATION PROBLEM ARISING IN HEMODYNAMICS
}

\author{
JOÃO JANELA \\ Instituto Superior de Economia e Gestão and CEMAT/IST, Universidade Técnica de Lisboa \\ Rua do Quelhas 6, 1200 Lisboa, Portugal \\ E-mail: jjanela@iseg.utl.pt
}

\section{ADÉLIA SEQUEIRA}

Instituto Superior Técnico and CEMAT/IST, Universidade Técnica de Lisboa

Av. Rovisco Pais 1, 1049-001 Lisboa, Portugal

E-mail: adelia.sequeira@math.ist.utl.pt

\begin{abstract}
Experimental evidence collected over the years shows that blood exhibits nonNewtonian characteristics such as shear-thinning, viscoelasticity, yield stress and thixotropic behaviour. Under certain conditions these characteristics become relevant and must be taken into consideration when modelling blood flow. In this work we deal with incompressible generalized Newtonian fluids, that account for the non-constant viscosity of blood, and present a new numerical method to handle fluid-rigid body interaction problems. The work is motivated by the investigation of interaction problems occurring in the human cardiovascular system, where the rigid bodies may be blood particles, clots, valves or any structure that we may assume to move rigidly. This method is based on a variational formulation of the fully coupled problem in the whole fluid/solid domain, in which constraints are introduced to enforce the rigid motion of the body and the equilibria of forces and stresses at the interface. The main feature of the method consists in introducing a penalty parameter that relaxes the constraints and allows for the solution of an associated unconstrained problem. The convergence of the solution of the relaxed problem is established and some numerical simulations are performed using common benchmarks for this type of problems.
\end{abstract}

2000 Mathematics Subject Classification: Primary 65M60; Secondary 74F10, 76Z05.

Key words and phrases: constrained minimization, hyper-viscosity method, fluid-rigid body interaction, shear-thinning fluid.

Research funded by the Center for Mathematics and its Applications - CEMAT through FCT funding program. The project HPRN-CT-2002-00270 (Research Training Network 'Haemodel' of the European Union) is also acknowledged.

The paper is in final form and no version of it will be published elsewhere. 
1. Introduction. The theoretical and numerical study of fluid-structure or fluid-particle interaction problems is of major importance when modelling several phenomena occuring in the human cardiovascular system, like the rolling of white blood cells (see [1]), or the motion and design of prostetic heart valves (see [9] ). There are also important industrial applications involving the settling and lift-off of particles in channel flows in the petroleum and coal industries (see $[2,7]$ and references therein).

The rheological characteristics of blood are determined by its complex morphology that we will briefly describe (for details see Sequeira and Janela [16], Robertson, Sequeira and Kameneva [15] and Robertson Sequeira and Owens [14]). Blood is a complex mixture consisting of different particles (erythrocytes, leukocytes, platelets and other matter) suspended in an aqueous polymer solution, the plasma (Newtonian fluid). These suspended particles, consisting mostly of erythrocytes (red blood cells) form about $45 \%$ of the volume of normal human blood and their effect should not be ignored. It is not feasible to track the individual behaviour of each RBC but it is possible to build homegenized models that reproduce the main non-Newtonian effects caused by their presence.

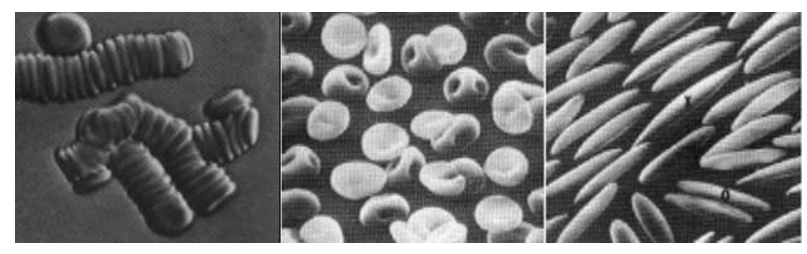

(a)

(b)

(c)

Fig. 1. Red blood cells at different shear rates (reprinted from Caro et al. [4])

The main non-Newtonian effects experimentally observed in blood are the shearthinning viscosity, the existence of a yield stress and viscoelasticity. At low shear rates blood has a high apparent viscosity, due to erythrocyte aggregation into clusters called rouleaux (see Figure 1 (a)), that can be long enough to prevent blood from flowing below a certain yield stress. As the shear rate increases, the cells become disaggregated (Figure $1(\mathrm{~b})$ ) and deform into an infinite variety of shapes and align with the direction of blood flow, resulting in a reduction of viscosity. Also, since blood cells are essentially elastic membranes filled with a fluid, it seems reasonable to expect blood to behave like a viscoelastic fluid, at least under certain conditions. The shear-thinning viscosity reduces velocity and increases shear rate in regions of constant shear stress while the viscoelasticity amplifies the effect of normal stresses in regions of constant shear-stress (see e.g. [11]).

In vessels of medium and large size $(0.4-2.5 \mathrm{~cm}$ diameter $)$ the typical values of the shear rate are high enough for the viscosity to be considered constant and therefore blood can be modelled as a Newtonian fluid. In smaller vessels, or more generally in regions of low shear rate, the effect of variable shear-thinning viscosity should not be neglected. In this work we will disregard the yield stress and viscoelastic effects and model blood as a shear-thinning generalized Newtonian fluid. In this idealized fluid, that we use as a model 
for blood, we will consider the motion of rigid particles that, as a first approximation, can mimic the motion of leukocytes or other transported cells in blood.

Numerical simulations of fluid-rigid body interactions can be carried out in different ways, roughly divided in two main classes. The first one involves a moving mesh following the moving part of the domain. In the second approach the whole computational domain is covered by a static mesh, leading to fictitious domain or embedded domain methods that make use of Lagrange multipliers to enforce the velocity in the solid phase. We elaborate on a method that falls into the second class, initially proposed by Janela, Maury and Lefevre [9] in the Newtonian framework and later extended to non-Newtonian fluids by Janela, Sequeira and Carapau [10]. The difference with respect to similar methods is the use of a penalty operator instead of Lagrange multipliers.

2. Continuous model problem. For simplicity, in the definition of the underlying mathematical and mechanical problem, we consider a connected, bounded and regular domain $\mathcal{O} \subset \mathbb{R}^{2}$ and define $B$ as a multiply connected regular set such that $\bar{B} \subset \mathcal{O}$. In particular we consider $B=\bigcup_{i=1}^{N} B_{i}$, where the sets $B_{i}, i=1, \ldots, N$, with boundary $\gamma_{i}$, are open and simply connected.

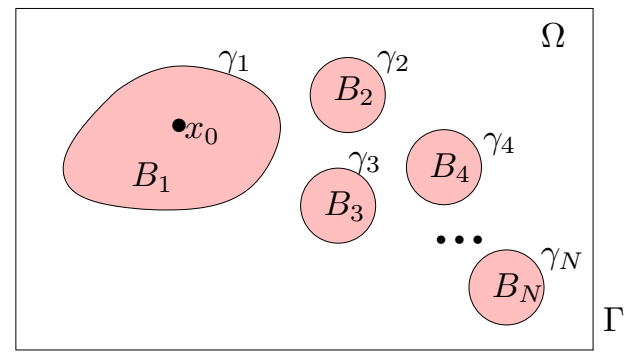

Fig. 2. Model domain

We assume that, at time $t \geq 0, \Omega=\mathcal{O}-\bar{B}$ is filled with a fluid and $B$ is a rigid inclusion (particle or particles) in $\mathcal{O}$. We denote by $\Gamma$ the boundary of $\mathcal{O}$ and by $\gamma=\cup_{i=1}^{N} \gamma_{i}$ the boundary of $B$. Since the position of $B$ is likely to vary over time, these sets will be referred to as $B_{t}$ and $\Omega_{t}$.

Rigid body motion. The motion of a body $\mathcal{B}_{i}$, occupying the set $B_{i}$, is said to be rigid if the velocity field over $B_{i}$ can be written as

$$
\boldsymbol{u}(\boldsymbol{x})=\mathbf{U}_{i}+\omega_{i} \times\left(\boldsymbol{x}-\mathbf{G}_{i}\right),
$$

where $\omega_{i}$ and $\mathbf{U}_{i}$ are respectively the angular and translational velocities and $\mathbf{G}_{i}$ is a point in $\mathcal{B}_{i}$, usually the center of mass. It can easily be shown that equation (1) is equivalent to

$$
\nabla \boldsymbol{u}+(\nabla \boldsymbol{u})^{T}=0, \quad \text { on } B_{i} .
$$

Moreover, for the purpose of coupling the body and fluid equations, condition (2) only needs to be enforced on the boundary of the body. We say that $\mathcal{B}=\cup \mathcal{B}_{i}$ is a system of rigid particles if condition 2 is satisfied for each connected component of $B$. 
Fluid motion. The conservation of linear momentum for the motion of a fluid with velocity $\boldsymbol{u}$ in $\Omega_{t}$ is given by

$$
\rho_{f}\left(\frac{\partial \boldsymbol{u}}{\partial t}+\boldsymbol{u} \cdot \nabla \boldsymbol{u}\right)=\nabla \cdot \boldsymbol{\sigma}+\boldsymbol{f}_{f}, \quad \text { in } \Omega_{t},
$$

where $\rho_{f}$ is the constant density, $\boldsymbol{f}_{f}$ the external body forces per unit volume (e.g. gravity) and $\boldsymbol{\sigma}$ is the Cauchy stress tensor. We also assume the incompressibility of the fluid and, as a consequence, the conservation of mass reduces to

$$
\nabla \cdot \boldsymbol{u}=0, \quad \text { in } \Omega_{t} .
$$

The Cauchy stress tensor for incompressible fluids can be split in terms of the hydrostatic pressure $p$ and the extra stress tensor $\tau$, i.e.

$$
\boldsymbol{\sigma}=-p \mathbf{I}+\boldsymbol{\tau}
$$

Finally, in order to close the system, we must specify a constitutive relation for the fluid. We start from the assumption that the fluid is incompressible and the Cauchy stress tensor only depends on the velocity gradient. This means that the current state of stress depends only on the current rate of deformation, and not on the history of deformations the fluid may have undergone in the past. Demanding invariance under a superposed rigid motion, we see that the most general form $\boldsymbol{\sigma}$ can have is

$$
\boldsymbol{\sigma}=\alpha \mathbf{I}+\phi_{1} \boldsymbol{D}+\phi_{2} \boldsymbol{D}^{2}
$$

where $\boldsymbol{D}(\boldsymbol{u}):=\left(\nabla \boldsymbol{u}+(\nabla \boldsymbol{u})^{T}\right) / 2$ is the symmetric part of the velocity gradient and $\phi_{1}, \phi_{2}$ are only functions of the principal invariants of $\boldsymbol{D}\left(I_{D}=\operatorname{tr}(\boldsymbol{D}), I I_{D}=\left(\operatorname{tr}(\boldsymbol{D})^{2}-\right.\right.$ $\left.\left.\operatorname{tr}\left(\boldsymbol{D}^{2}\right)\right) / 2, I I I_{D}=\operatorname{det}(\boldsymbol{D})\right)$. Fluids described by the constitutive equation (6) are known as incompressible Reiner-Rivlin fluids.

Further assuming that $\phi_{2}=0$ (this is supported by experimental measurements obtained from a wide variety of fluids) and that the dependence of $\phi_{1}$ on $I I I_{D}$ is negligible, we finally obtain the generalized Newtonian fluids. For these fluids the constitutive equation (5) allows for the explicit calculation of $\boldsymbol{\sigma}$ since the extra stress tensor is written in terms of $\boldsymbol{D}$ and of the shear rate $\dot{\gamma}$, defined by

$$
\dot{\gamma}=\sqrt{2 \mathbf{D}: \mathbf{D}}=\sqrt{\sum_{i, j} 2 D_{i j} D_{i j}}=\sqrt{2 \sum_{i}\left(\frac{\partial u_{i}}{\partial x_{i}}\right)^{2}+\sum_{i \neq j}\left(\frac{\partial u_{i}}{\partial x_{j}}+\frac{\partial u_{j}}{\partial x_{i}}\right)^{2}}
$$

this leads to the following form of the constitutive equation

$$
\boldsymbol{\tau}=2 \eta(\dot{\gamma}) \boldsymbol{D}(\boldsymbol{u})=\eta(\dot{\gamma})\left(\nabla \boldsymbol{u}+(\nabla \boldsymbol{u})^{T}\right) .
$$

If in equation (7) $\eta$ is taken as a constant, the classical Navier-Stokes equations are recovered. The precise functional form of $\eta$ is not derived from any theoretical principle, but chosen to fit experimental data. Over the years many such models have been proposed in order to fit experimental data from several types of fluids. In most of these models the viscosity is considered to be a monotone, bounded function of shear rate, usually written in the form

$$
\eta(\dot{\gamma}):[0,+\infty] \rightarrow\left[\eta_{0}, \eta_{\infty}\right], \quad \dot{\gamma} \mapsto \eta_{\infty}+\left(\eta_{0}-\eta_{\infty}\right) F(\dot{\gamma})
$$


where $\eta_{0}$ and $\eta_{\infty}$ are the asymptotic viscosities at zero and infinite shear rates, respectively and $F(\cdot)$ is a bounded monotone function such that

$$
\lim _{\dot{\gamma} \rightarrow 0} F(\dot{\gamma})=1, \quad \lim _{\dot{\gamma} \rightarrow+\infty} F(\dot{\gamma})=0 .
$$

There are many usual choices for this function $F$, expressing how the transition between the high and low shear rate viscosity approximations is made. In this work, since we aim at applications to hemodynamics, we use the Carreau-Yasuda viscosity model which fits well experimental measurements of blood (see [5] and references therein) and, for shear-thinning fluids, is given by

$$
F(\dot{\gamma})=\left(1+(\lambda \dot{\gamma})^{a}\right)^{\frac{n-1}{a}} .
$$

This is a five constants model where, apart from $\eta_{0}$ and $\eta_{\infty}$, we have a relaxation time $\lambda$, and parameters $a, n$ chosen to fit experiments (when $a=2$ this is known as the Carreau model). When $n=1$ or $a=0$ or $\lambda=0$ the viscosity is constant and the fluid behaves as Newtonian. For $n>1$ the fluid is shear-thickening and for $n<1$ it has shear-thinning behaviour. Figure 3 illustrates the viscosity behaviour for several values of the power index $n$.

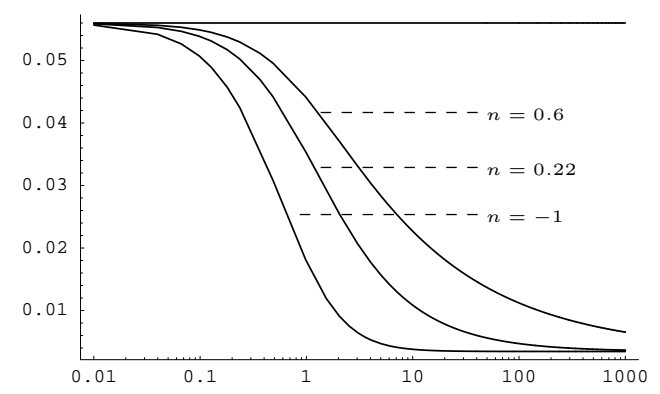

Fig. 3. Viscosity vs. shear rate for the Carreau-Yasuda model, at different values of the power index $(n=0.22$ corresponds to blood, see [5]).

The coupled problem. In order to couple the solid and fluid phases we must prescribe compatibility conditions consisting in instantaneous equilibria of forces exerted in the fluid/body interface. Denoting by $\zeta_{i}(t)$ the position of the geometrical center of $B_{i}$, we must have

$$
\begin{aligned}
M_{i} \zeta_{i}^{\prime \prime}(t) & =-\int_{\gamma_{i}} \boldsymbol{\sigma} \cdot \boldsymbol{n}+\int_{B_{i}} \boldsymbol{f}_{b}, \quad i=1, \cdots, N \\
J_{i} \omega_{i}^{\prime}(t) & =-\int_{\gamma_{i}}\left(\boldsymbol{x}-\zeta_{i}(t)\right)^{\perp} \cdot \boldsymbol{\sigma} \boldsymbol{n}+\int_{B_{i}}\left(\boldsymbol{x}-\zeta_{i}(t)\right)^{\perp} \boldsymbol{f}_{b},
\end{aligned}
$$

where $M_{i}$ and $J_{i}$ are the (constant) mass and momentum of inertia of $B_{i}$, given by $M_{i}=\int_{B_{i}} \rho_{b}$ and $J_{i}=\int_{B_{i}}=\rho_{b}\left|\boldsymbol{x}-\boldsymbol{x}_{0}\right|^{2}, \rho_{b}$ is the density of the body and $\boldsymbol{f}_{b}$ are the external body forces exerted on $B_{t}$.

The fully coupled fluid-rigid body interaction problem consists in finding $\boldsymbol{u}, p, \omega_{i}$ and $\mathbf{U}_{i}$ such that 


$$
\begin{cases}\rho_{f} \frac{D \boldsymbol{u}}{D t}=\nabla \cdot(-p \mathbf{I}+\eta(\dot{\gamma}) \mathbf{D}(\mathbf{u}))+\boldsymbol{f} & \text { in } \Omega_{t}, \\ \nabla \cdot \boldsymbol{u}=0 & \text { on } \Gamma, \\ \boldsymbol{u}=0 & \text { on } \gamma, \\ \nabla \boldsymbol{u}+(\nabla \boldsymbol{u})^{T}=0 & i=1, \ldots, N \\ M_{i} \zeta_{i}^{\prime \prime}(t)=-\int_{\gamma_{i}} \boldsymbol{\sigma} \cdot \boldsymbol{n}+\int_{B_{i}} \boldsymbol{f}_{b}, & i=1, \ldots, N . \\ J_{i} \omega_{i}^{\prime}(t)=-\int_{\gamma_{i}}\left(\boldsymbol{x}-\zeta_{i}(t)\right)^{\perp} \cdot \boldsymbol{\sigma n}+\int_{B_{i}}\left(\boldsymbol{x}-\zeta_{i}(t)\right)^{\perp} \boldsymbol{f}_{b} & \end{cases}
$$

We remark that the constitutive equation for the stress tensor does not need to be of this particular form and, as long as $\tau$ can be computed, more complex fluids (e.g. viscoelastic fluids) can be considered without any changes in our method.

Having in mind the discretization by finite elements, we write the problem in a weak form. Let us consider the function spaces $V=\left[H_{0}^{1}(\mathcal{O})\right]^{2}$ and $Q=\left\{q \in L^{2}(\mathcal{O}): \int_{\Omega} q=0\right\}$. Multiplying the fluid equations by test functions $\boldsymbol{v} \in V$ and $q \in Q$ and integrating by parts over $\Omega$ we obtain

$$
\begin{gathered}
\int_{\Omega} \rho_{f} \frac{D \boldsymbol{u}}{D t} \cdot \boldsymbol{v}+\int_{\Omega} \boldsymbol{\tau}: \nabla \boldsymbol{v}-\int_{\Omega} p \nabla \cdot \boldsymbol{v}=\int_{\Omega} \boldsymbol{f} \cdot \boldsymbol{v}+\int_{\partial \Omega} \boldsymbol{v} \cdot(\boldsymbol{\sigma} \cdot \mathbf{n}), \quad \forall \boldsymbol{v} \in V, \\
\int_{\Omega} q \nabla \cdot \boldsymbol{u}=0, \quad \forall q \in Q .
\end{gathered}
$$

We now consider the space of rigid motions over $B, K_{B}=\left\{\boldsymbol{v} \in V: \nabla \boldsymbol{v}+(\nabla \boldsymbol{v})^{T}=0\right.$ in $B$, take into account the compatibility and boundary conditions, and rewrite the variational formulation introduced above as follows: for every $t>0$, find $\boldsymbol{u} \in K_{B}$ and $p \in Q$,

$$
\left\{\begin{array}{l}
\int_{\mathcal{O}} \rho \frac{D \boldsymbol{u}}{D t} \cdot \boldsymbol{v}+\int_{\mathcal{O}} \boldsymbol{\tau}: \nabla \boldsymbol{v}=\int_{\mathcal{O}} p \nabla \cdot \boldsymbol{v}+\int_{\mathcal{O}} \boldsymbol{v} \cdot \boldsymbol{f}, \quad \forall \boldsymbol{v} \in K_{B}, \\
\int_{\mathcal{O}} q \nabla \boldsymbol{u}=0, \quad \forall q \in Q,
\end{array}\right.
$$

where $\rho=\rho_{f} \chi_{\Omega}+\rho_{b}\left(1-\chi_{\Omega}\right)$ and $\boldsymbol{f}$ is extended by zero outside $B$. We observe that formulation (13) does not contain any boundary terms.

3. Discretization in time. The method of characteristics is used to discretize the material time derivative. If we denote by $\mathbf{X}^{n}(x)$ the characteristic associated with $\boldsymbol{u}$ then, at time step $n+1$, we have

$$
\frac{\partial \boldsymbol{u}^{n+1}}{\partial t}+\boldsymbol{u}^{n+1} \cdot \nabla \boldsymbol{u}^{n+1} \approx \frac{1}{\Delta t}\left(\boldsymbol{u}^{n+1}-\boldsymbol{u}^{n} \circ \mathbf{X}^{n}\right) \approx \frac{1}{\Delta t}\left(\boldsymbol{u}^{n+1}-\boldsymbol{u}^{n}\left(\boldsymbol{x}-\boldsymbol{u}^{n}(\boldsymbol{x}) \Delta t\right)\right) .
$$

The resulting problem is still not linear since the calculation of $\dot{\gamma}$, necessary to compute the viscosity at each time step, involves the velocity in a nonlinear way. To avoid this problem we consider a semi-implicit approximation of the extra stress tensor,

$$
\boldsymbol{\tau}^{n+1}=2 \eta\left(\dot{\gamma}^{n+1}\right) \mathbf{D}\left(\boldsymbol{u}^{n+1}\right) \approx 2 \eta\left(\dot{\gamma}^{n}\right) \mathbf{D}\left(\boldsymbol{u}^{n+1}\right) .
$$


Considering the above approximations, the discretized problem reads: find $\boldsymbol{u}^{n+1} \in K_{B^{n+1}}$ and $p \in L_{0}^{2}(\mathcal{O})$ such that

$$
\begin{aligned}
\frac{1}{\Delta t} \int_{\mathcal{O}} \rho^{n+1} \boldsymbol{u}^{n+1} \cdot \boldsymbol{v}+\int_{\mathcal{O}} 2 \eta\left(\dot{\gamma}^{n}\right) \mathbf{D}\left(\boldsymbol{u}^{n+1}\right): \nabla \boldsymbol{v}-\int_{\mathcal{O}} p^{n+1} \nabla \cdot \boldsymbol{v} \\
\quad=\frac{1}{\Delta t} \int_{\mathcal{O}}\left(\rho^{n} \boldsymbol{u}^{n}\right) \circ \mathbf{X}^{n} \cdot \boldsymbol{v}+\int_{\mathcal{O}} \boldsymbol{f}^{n+1} \cdot \boldsymbol{v}, \quad \forall \boldsymbol{v} \in K_{B^{n+1}}
\end{aligned}
$$

and

$$
\int_{\mathcal{O}} q \nabla \cdot \boldsymbol{u}^{n+1}=0, \quad \forall q \in Q
$$

where, knowing $\boldsymbol{u}^{n}, B^{n+1}$ is computed updating the position of $B^{n}$, i.e. $B^{n+1}$ is the rigid body at time $t_{n+1}$. In fact, it is easier to use $\mathbf{U}^{n}$ and $\omega^{n}$, that can be computed from $\boldsymbol{u}^{n}$. When $B$ is a circle of radius $r$, we have the following formulas for $\mathbf{U}$ and $\omega$

$$
\begin{aligned}
U_{i} & =\frac{1}{\pi r^{2}} \int_{B} u_{i}, \quad i=1,2, \\
\omega & =-\frac{2}{\pi r^{4}} \int_{B}(\boldsymbol{u}-\mathbf{U}) \times(\boldsymbol{x}-\mathbf{G}) .
\end{aligned}
$$

If we make no assumptions on the shape of the body, $\omega$ and $\mathbf{U}$ can still be computed by solving the linear system

$$
\begin{cases}|B| U_{1}+\alpha_{2} \omega & =\int_{B} u_{1}, \\ |B| U_{2}-\alpha_{1} \omega & =\int_{B} u_{2}, \\ -\alpha_{2} U_{1}+\alpha_{1} U_{2}-\alpha_{3} \omega & =\beta,\end{cases}
$$

where $\alpha_{1}=\int_{B}\left(x_{1}-G_{1}\right), \alpha_{2}=\int_{B}\left(x_{2}-G_{2}\right), \alpha_{3}=\int_{b}\|\boldsymbol{x}-\mathbf{G}\|^{2}$ and $\beta=\int_{B}\left(\left(x_{1}-G_{1}\right) u_{2}\right.$ $\left.-\left(x_{2}-G_{2}\right) u_{1}\right)$. This problem is equivalent to the minimization over $K_{B^{n+1}}$ of the functional

$$
J^{n}(\boldsymbol{v})=\frac{1}{2 \Delta t} \int_{\mathcal{O}}\left(\rho^{n+1} \boldsymbol{v}^{2}+2\left(\rho^{n} \boldsymbol{u}^{n}\right) \circ \mathbf{X} \cdot \boldsymbol{v}\right)+\int_{\mathcal{O}}\left(2 \eta\left(\dot{\gamma}^{n}\right) \mathbf{D}(\boldsymbol{v}): \nabla \boldsymbol{v}-\boldsymbol{f}^{n+1} \cdot \boldsymbol{v}\right)
$$

and it will be solved within the theoretical framework presented in the next section. This kind of time discretization corresponds to a non-centered finite-difference method along characteristics and falls within the class of Lagrange-Galerkin methods used to treat convection dominated diffusion equations. At least in the Newtonian case, this method of characteristics has been proven to be of order 2 in time [3]. In our case, requiring enough regularity on the viscosity law, the same result should hold, but this is the object of current investigation.

At each time step the problem must be discretized in space. This will be done using a Petrov-Galerkin method based on a suitable meshing of the computational domain (fluid + solid). The conformal finite element spaces chosen to approximate the unknowns are $P^{2}$ for the velocity field and $P^{1}$ for the pressure. This combination of spaces satisfies the discrete inf-sup or LBB stability condition.

4. Abstract penalty method. The weak formulation (13) is called constrained since the enforcement of rigid motion over $B$ is embedded in the functional spaces. The pe- 
nalization approach aims at relaxing this constraint which can be done in an abstract setting. We next summarize the main results introduced in [12].

Let $V$ be a Hilbert space with inner product $(\cdot, \cdot), a(\cdot, \cdot)$ a symmetric, bilinear continuous and coercive form and $\varphi \in V^{\prime}$. Let us also define the functional $J(v)=\frac{1}{2} a(v, v)-$ $\langle\varphi, v\rangle$. The proof of the following propositions is straightforward (see [13]).

Proposition 1. Let $K$ be a subspace of $V$. The constrained minimization problem

$$
(P)\left\{\begin{array}{l}
\text { Find } u \in K \text { such that } \\
J(u)=\inf _{v \in K} J(v)
\end{array}\right.
$$

has a unique solution characterized by

$$
a(u, v)=\langle\varphi, v\rangle, \quad \forall v \in K,
$$

and the following estimate holds

$$
|u| \leq \frac{1}{\alpha}\|\varphi\| .
$$

Proposition 2. Let $H$ be a Hilbert space and $J: H \rightarrow \mathbb{R} \cup\{\infty\}$ a convex functional, not identically infinite. For every sequence $\left(x_{n}\right)$ weakly convergent to $x \in H$ we have $J(x) \leq \liminf J\left(x_{n}\right)$.

Consider now a bilinear, symmetric and positive form $b(\cdot, \cdot)$ and its kernel $K=$ $\{v \in V: b(v, v)=0\}$. For all $\varepsilon>0$ we consider the unconstrained minimization problem $\left(P_{\varepsilon}\right)$ of the functional

$$
J_{\varepsilon}(v)=J(v)+\frac{1}{\varepsilon} b(v, v),
$$

that has also a unique solution $u^{\varepsilon} \in V$. The following result holds

THEOREM 3. The sequence $\left(u^{\varepsilon}\right)$ of solutions of problem $\left(P_{\varepsilon}\right)$ converges to the solution $u$ of problem $(P)$, in $V$.

Proof. Writing the variational formulation of $\left(P_{\varepsilon}\right)$, using in particular $v=u^{\varepsilon}$ and considering the properties of $a(\cdot, \cdot)$ and $b(\cdot, \cdot)$ we get

$$
a\left(u^{\varepsilon}, u^{\varepsilon}\right) \leq C_{0}+C_{1}\left|u^{\varepsilon}\right| \Rightarrow \alpha\left|u^{\varepsilon}\right|^{2} \leq C_{0}+C_{1}\left|u^{\varepsilon}\right|
$$

which shows that the sequence $\left(u^{\varepsilon}\right)$ is bounded and therefore there is a subsequence converging weakly to $z \in V$. Using the positivity of $b(\cdot, \cdot)$ and the definition of $u^{\varepsilon}$ we can see that $J\left(u^{\varepsilon}\right) \leq J_{\varepsilon}\left(u^{\varepsilon}\right) \leq J_{\varepsilon}(u)=J(u)$ and, using Proposition 2, we also have $J(z) \leq \lim \inf J\left(u^{\varepsilon}\right) \leq J(u)$.

It now suffices to show that $z$ verifies the constraint and can be identified with the solution of problem $(\mathrm{P})$. We start by showing that $b\left(u^{\varepsilon}, u^{\varepsilon}\right) \rightarrow 0$. Since $J_{\varepsilon}\left(u^{\varepsilon}\right) \leq J(u)$, we have

$$
\frac{1}{\varepsilon} b\left(u^{\varepsilon}, u^{\varepsilon}\right) \leq J(u)+\|\varphi\|_{V^{\prime}}\left|u^{\varepsilon}\right| \leq C
$$

and therefore $b\left(u^{\varepsilon}, u^{\varepsilon}\right) \rightarrow 0$ which, together with $0 \leq b(z, z) \leq \lim \inf b\left(u^{\varepsilon}, u^{\varepsilon}\right)$ means that $z \in K$ (because $b(z, z)=0$ ) and consequently $z=u$. The same argument can be applied to any convergent subsequence and so we prove weak convergence of $u^{\varepsilon}$ to $u$.

The proof of strong convergence is straightforward using the norm associated to the scalar product $(v, w)_{a}=a(v, w)$ (equivalent to the initial norm). 
Proposition 4. Denoting by $C$ and $\alpha$ the continuity and coercivity constants of $a(\cdot, \cdot)$, the following estimate holds:

$$
\left|u^{\varepsilon}-u\right| \leq \sqrt{\frac{C}{\alpha}} \operatorname{dist}\left(u^{\varepsilon}, K\right) .
$$

Only making further assumptions on $b(\cdot, \cdot)$ we can obtain a more usable error estimate for the penalized solution. The following proposition gives sufficient conditions for linear convergence.

Proposition 5. If we further assume that $b(\cdot, \cdot)$ is of the form $b(u, v)=(\Psi u, \Psi v)$, with $\Psi$ a linear, continuous functional with closed range over a Hilbert space $\Lambda$, there exists a constant $C>0$ such that

$$
\left|u^{\varepsilon}-u\right| \leq C \varepsilon
$$

Hyper-viscosity unconstrained formulation. The abstract penalty method already introduced can be applied to problem (13), recognizing that the space $K_{B}$ is the kernel of the operator

$$
b(\boldsymbol{u}, \boldsymbol{v})=\int_{B}\left(\nabla \boldsymbol{u}+(\nabla \boldsymbol{u})^{T}\right):\left(\nabla \boldsymbol{v}+(\nabla \boldsymbol{v})^{T}\right) .
$$

Instead of solving (13) in the constrained space $K_{B}$, we will solve the penalized unconstrained problem:

For every $t>0$, find $\boldsymbol{u} \in K_{B}, p \in Q$ :

$$
\begin{aligned}
\int_{\Omega} \rho \frac{D \boldsymbol{u}}{D t} \cdot \boldsymbol{v} & =\int_{\Omega}(\boldsymbol{\tau}: \nabla \boldsymbol{v}+p \nabla \boldsymbol{v}+\boldsymbol{f} \cdot \boldsymbol{v})-\frac{1}{\varepsilon} \int_{B} D \boldsymbol{u}: D \boldsymbol{v}, \quad \forall \boldsymbol{v} \in V, \\
\int_{\Omega} q \nabla \boldsymbol{u} & =0, \quad \forall q \in Q,
\end{aligned}
$$

that is equivalent to the unconstrained minimization of the functional

$$
J_{\varepsilon}^{n}(\boldsymbol{v})=J^{n}(\boldsymbol{v})+\int_{B} D \boldsymbol{v}: D \boldsymbol{v}
$$

Convergence when $\varepsilon \rightarrow 0$ is guaranteed by Proposition 5 . The main advantage of relaxing the rigid motion constraint is the possibility of using standard fluid solvers (with minor changes), avoiding heavy programming tasks. The name hyper-viscosity arises from the fact that, in the Newtonian case, the penalty term is the stiffness operator restricted to $B$ and multiplied by a factor of $1 / \varepsilon$. Since the leading coefficient of the global stiffness operator is the viscosity, the application of this method results in an increase of the viscosity over the $B$, by a factor of $1 / \varepsilon$.

5. Numerical results. The hyper-viscosity method was implemented using a general finite element solver/programming language called Freefem ++ (see [8]). The code accepts arbitrary 2D geometries for the domain and the body. We will present simple numerical simulations that test the angular and translational velocities reported by the method. The parameters used in the simulation are those appropriate to model blood: $\eta_{0}=0.056 \mathrm{~Pa} s$, $\eta_{\infty}=0.00345 \mathrm{Pas}, \lambda=1.1902 s, a=1.25$ and $n=0.22$ (see e.g. [5]). With the purpose of validating the method we test separately the rotation and translation of the rigid body. 


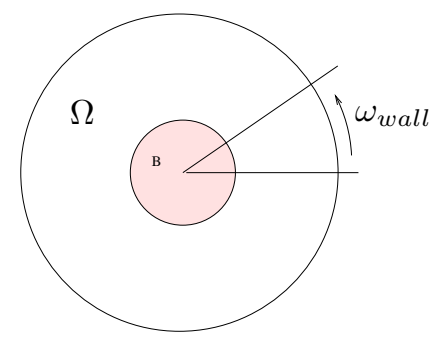

Fig. 4. Model problem for testing the pure rotation

Pure rotation motion. In this simulation the computational domain is a circle of radius $3 \mathrm{~cm}$ and the particle is a circle of unit radius, initially placed at the center of the domain. This is the $2 \mathrm{D}$ analog of flow between concentric cylinders. At time $t=0$ the outer boundary is set to move at constant angular velocity. Due to the no-slip boundary condition, the fluid starts a rotating movement to follow the outer boundary, also inducing the motion of the particle. The particle is initially at rest and starts an increasingly faster rotation until it reaches a terminal angular speed $\omega<\omega_{\text {wall }}$, without any translation. In figure 5 we show the time evolution of $\omega$ for different values of the power index $n$. The thicker line corresponds to the Newtonian fluid $(n=1)$. Above this curve we find the curves obtained for shear-thickening fluids $(n>1)$ and below those obtained for shear-thinning fluids $(n<1)$.

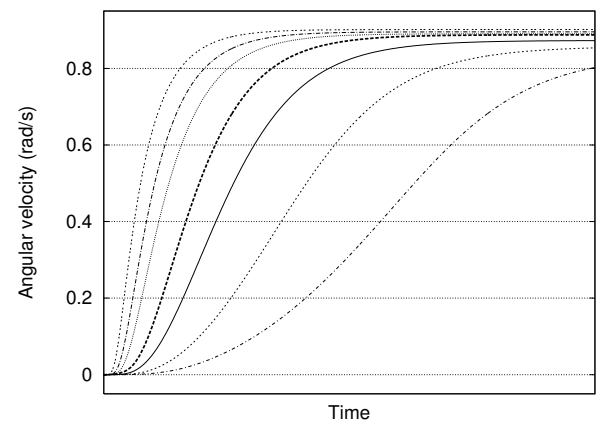

Fig. 5. Angular velocity for several values of the power index $(n=2.2,1.8,1.5,1,0.5,0,-0.5$, from left to right)

We observe that for all values of the power index the same terminal angular velocity is achieved for the particle. However, the higher is the power index, the faster is the convergence. The curves in figure 5 can be used to design a simple Couette viscometer to identify the power index of the fluid.

In figure 6 we observe that, apart from rotating, the body also undergoes a translation, describing a small orbit around the center of the domain which is due to numerical instabilities. This problem becomes relevant after a relatively large number of time steps, but can be arbitrarily delayed using finer meshes and smaller time steps.

Finally, figures 7 and 8 show plots illustrating the evolution of the streamlines and shear-dependent viscosity, from the transient to the steady-state period. We observe 


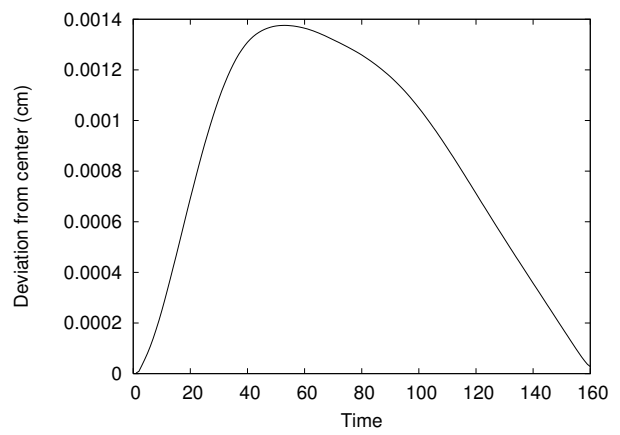

Fig. 6. Distance from the center of the particle to the center of the domain

that the streamlines, initially concentrated near the outer boundary, become equally distributed when the steady state is reached, which is due to the constant angular velocity attained by the whole fluid. The same behaviour occurs with the shear-dependent viscosity.

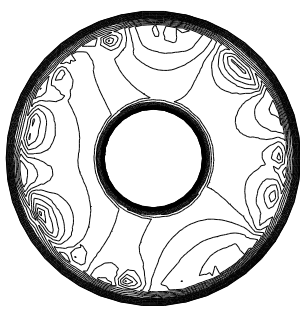

$t=0.1 \mathrm{~s}$

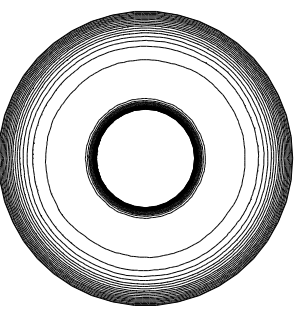

$t=1.5 \mathrm{~s}$

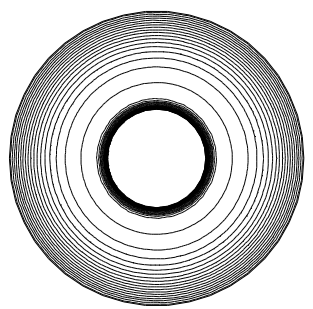

$t=4 s$

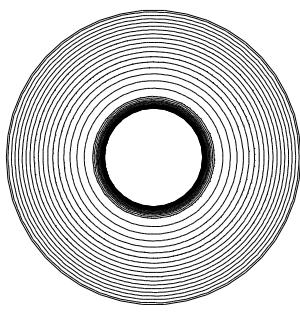

$t=20 s$

Fig. 7. Time evolution of the streamlines

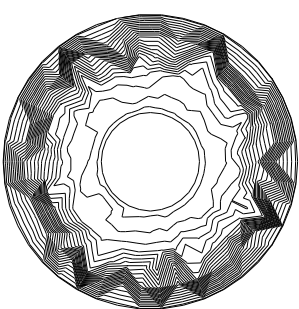

$t=0.1 s$

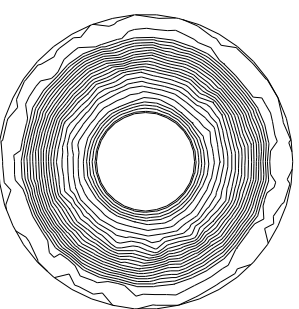

$t=1.5 \mathrm{~s}$

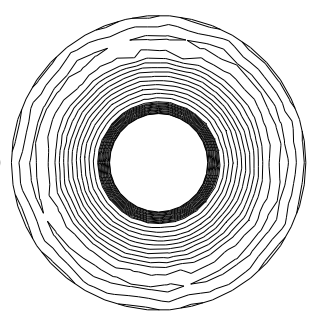

$t=4 s$

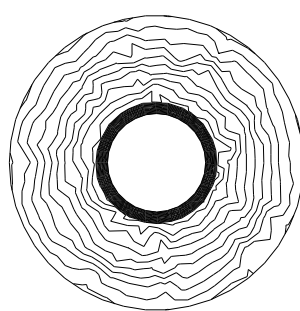

$t=20 s$

Fig. 8. Time evolution of the shear-dependent viscosity

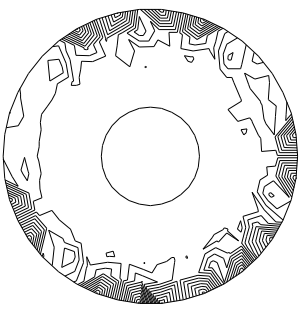

$t=0.1 s$

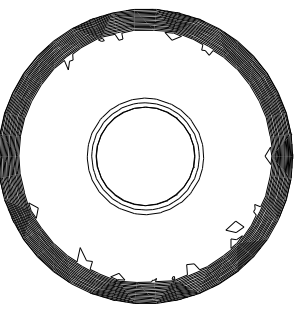

$t=1.5 \mathrm{~s}$

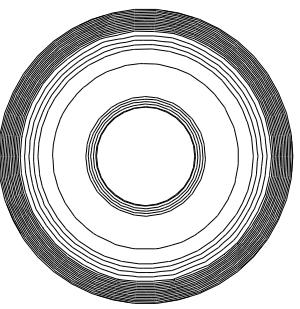

$t=4 s$

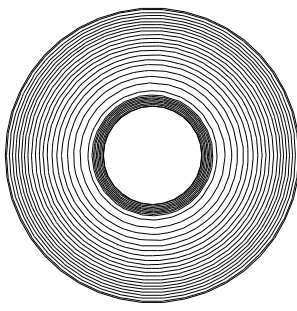

$t=20 \mathrm{~s}$

Fig. 9. Time evolution of the pressure 
Pure translation motion. Now the computational domain is a rectangular container $20 \mathrm{~cm}$ high and $10 \mathrm{~cm}$ wide, filled with the same fluid as in the previous example, that is, $\mathcal{O}=] 0,10[\times] 0,20[$. At time $t=0$ a particle (circle of radius 1 ) is dropped from the initial position $(5,15)$ and falls under the action of gravity. More precisely, the resultant of applied volumic forces $\boldsymbol{f}$ combines gravity and buoyancy

$$
\boldsymbol{f}=|B| \rho_{b} \chi_{b} \mathbf{g}-|B| \rho_{b} \chi_{b} \mathbf{g} .
$$

The behaviour of this system is well known and, for constant viscosity, is discussed in any elementary physics book. There is a competition between gravity and the drag or viscous forces, the latter increases with the velocity of the body until it balances gravity and the body reaches a terminal velocity.

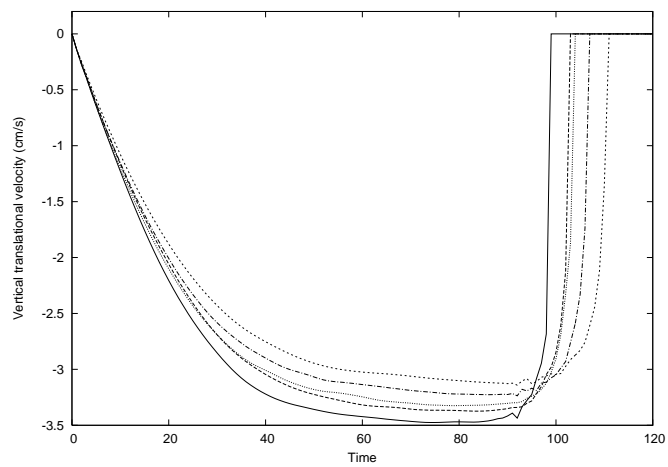

Fig. 10. Time evolution of the vertical translational velocity of the particle for several values of the power index $(n=0,0.5,1,1.5,2)$

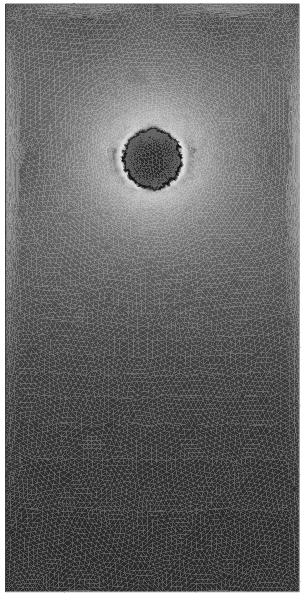

$t=0.5 \mathrm{~s}$

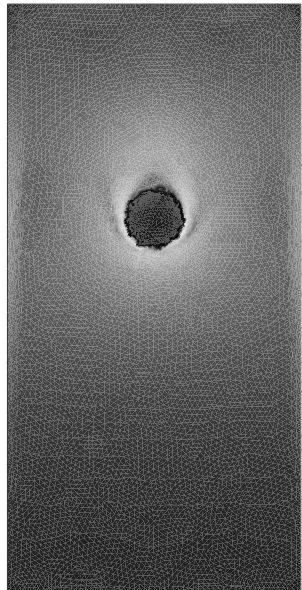

$t=1.5 \mathrm{~s}$

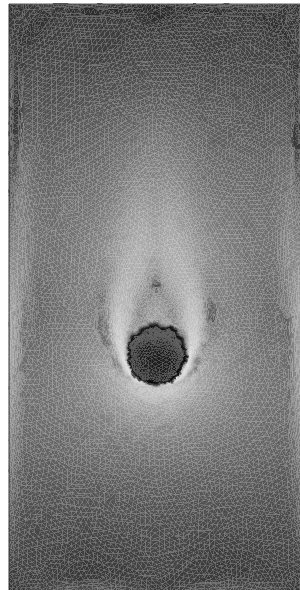

$t=3 \mathrm{~s}$

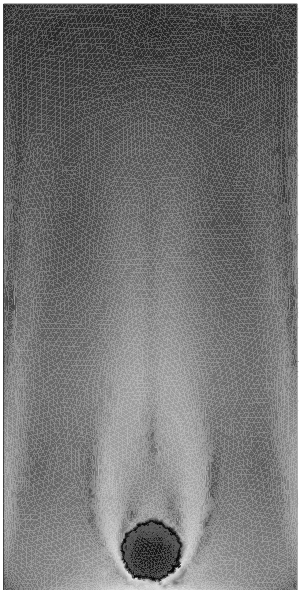

$t=5 s$

Fig. 11. Time evolution of the shear-dependent viscosity

In figure 10 we plot the velocity of the particle versus time. Velocity is zero in the beginning of the simulation (initially the body is at rest), then it decreases until a plateau 


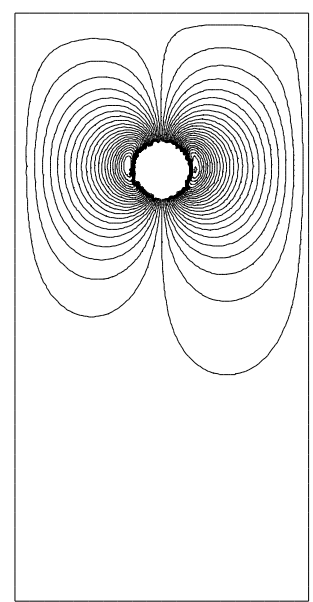

$t=0.5 \mathrm{~s}$

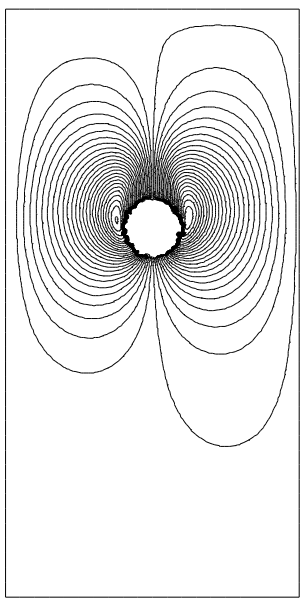

$t=1.5 \mathrm{~s}$

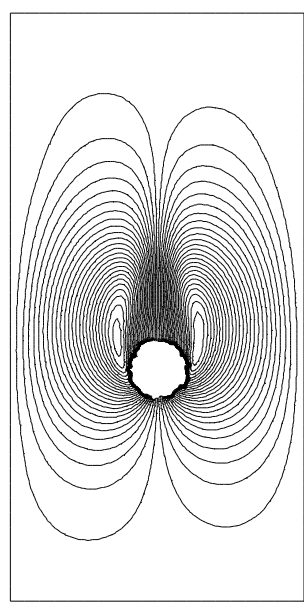

$t=3 \mathrm{~s}$

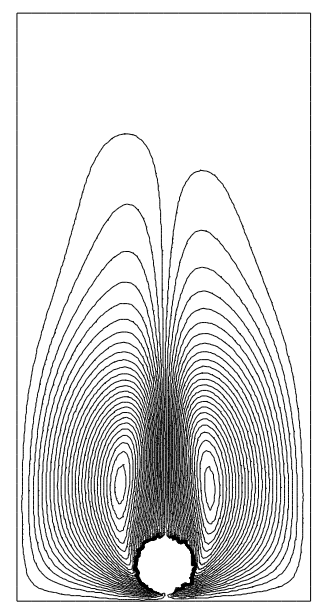

$t=5 s$

Fig. 12. Time evolution of the velocity streamlines

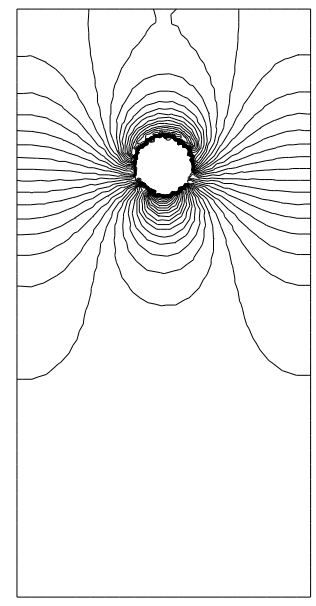

$t=0.5 s$

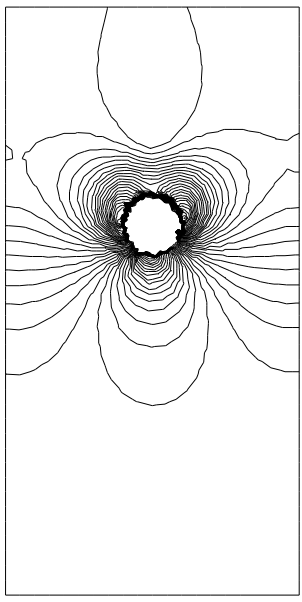

$t=1.5 \mathrm{~s}$

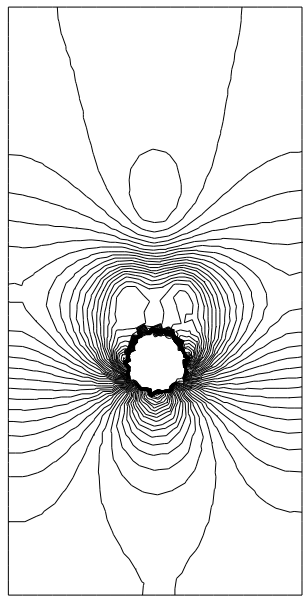

$t=3 \mathrm{~s}$

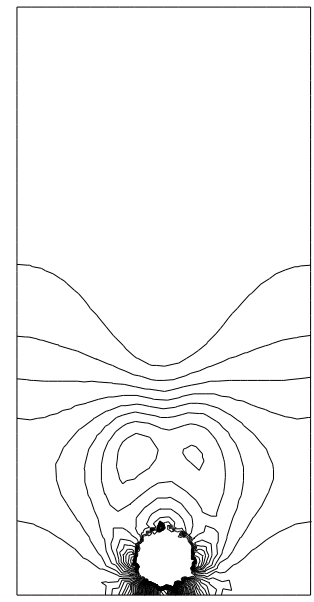

$t=5 s$

Fig. 13. Time evolution of the pressure isolines

is attained and would remain constant if the container would be infinite, finally going abruptly to zero when the body touches the bottom. This is the general qualitative behaviour. When varying the power index, different terminal velocities are obtained. This is easily explained by the fact that the drag force is proportional to velocity.

In the shear-thinning case, as the velocity of the particle increases, the shear rate will be higher around the particle, the viscosity decreases, and so does the drag force, allowing for velocity to increase even further. The balance between gravity and drag will appear only after viscosity reaches its lower bound, $\eta_{\infty}$.

In the shear-thickening case, exactly the opposite happens. As velocity increases, shear rate will be higher around the particle and the viscosity increases, together with the drag 
force. In this way the balance between gravity and drag force arises sooner in time and lower (in absolute value) terminal velocities are obtained.

In figures 11 and 12 we display the shear-dependent viscosity and the streamlines in selected moments of the simulation. In the viscosity plot it is clearly seen the wake left behind by the particle. In figure 13 we present the pressure isolines.

6. Conclusions. It has been shown that the proposed penalty or hyper-viscosity method is well suited for the simulation of fluid - rigid body interaction problems, in the case of a generalized Newtonian fluid. The convergence of the penalization procedure has been proved and numerical tests were carried out, showing differences between the Newtonian and non-Newtonian fluid behaviour, visible in the computation of both angular and translational velocities.

The main advantage of this method is the possibility of using, with minor modifications, widely available finite element packages and solvers for the Navier-Stokes equations. The implementation of this new method only requires the introduction of shear-dependent viscosity and very limited changes in the stiffness matrix.

The proof of convergence of the numerical method is the subject of current investigation, since until now it was only proven the convergence of the exact solutions of the penalized problems to the exact solution of the coupled problem. The main difficulty so far is a competitive behaviour between the penalty parameter $\varepsilon$ and the space discretization parameter $h$. Compatible choices of these parameters are required to ensure convergence of the numerical method and this seems to depend on the geometrical properties of the domain.

Future work includes the derivation of sharp error estimates, explaining the observed rates of convergence. The extension of this method to viscoelastic models like Oldroyd-B or shear-dependent Oldroyd-B fluids is also foreseen and should not pose any additional difficulties, apart from the ones usually experienced for higher Weissenberg numbers.

\section{References}

[1] A. M. Artoli, A. Sequeira, A. S. Silva and C. Saldanha, Leukocyte rolling and recruitment by endothelial cells: hemorheological experiments and numerical simulations, Journal of Biomechanics (2007), submitted.

[2] R. Bai, R. Glowinski, D. D. Joseph and T.-W. Pan, Direct simulation of the motion of settling ellipsoids in a Newtonian fluid, in: Proc. of the 14th Int. Conf. on Domain Decomposition Methods, 2002.

[3] M. Bensaada, D. Esselaoui, P. Saramito and A. Sequeira, Noncentered second-order Galerkin-Lagrange method for the transient Navier-Stokes equations, submitted.

[4] C. G. Caro, T. J. Peddley, R. C. Schroter and W. A. Seed, The Mechanics of the Circulation, Oxford University Press, Oxford, 1978.

[5] Y. I. Cho and K. R. Kensey, Effects of the non-Newtonian viscosity of blood on flows in diseased arterial vessel. Part I: Steady Flows, Biorheology 28 (1991), 241-262.

[6] R. Glowinski and B. Maury, Fluid-particle flows: a symmetric formulation, C. R. Acad. Sci. Paris Sér. I 324 (1997), 1079-1084. 
[7] P. Y. Huang, T. Ko, D. D. Joseph, and N. A. Patankar, Lift-off of a single particle in Newtonian and viscoelastic fluids by direct numerical simulation, Journal of Fluid Mechanics 438 (2001), 67-100.

[8] F. Hecht, A. Le Hyaric, O. Pironneau and K. Ohtsuka, Freefem ++ version 2.3-3, Laboratoire Jacques-Louis Lions, Université Pierre et Marie Curie, Paris (http://www.freefem.org), 2006.

[9] J. Janela, A. Lefebvre and B. Maury, A penalty method for the simulation of fluid-rigid body interaction, ESAIM Proceedings 14 (2005), 115-123.

[10] J. Janela, A. Sequeira and F. Carapau, A hyper-viscosity method for the interaction of a shear dependent fluid with a rigid body, WSEAS Transactions on Mathematics 4 (2006), Volume 5, 366-373.

[11] D. D. Joseph, Flow induced micro-structure in Newtonian and viscoelastic fluids, in: Proc. of the 5th World Congress of Chem. Eng., Particle Technology Track, AIChE 6 (1996), $3-16$.

[12] B. Maury, Optimisation sous contrainte et méthode des éléments finis, Université ParisSud, 2004.

[13] A. Quarteroni and A. Valli, Numerical Approximation of Partial Differential Equations, Springer Series in Computational Mathematics, Springer, Berlin, 1994.

[14] A. M. Robertson, A. Sequeira and R. G. Owens, Rheological models for blood, in: Cardiovascular Mathematics, A. Quarteroni et al. (eds.), Springer, 2007, to appear.

[15] A. M. Robertson, A. Sequeira and M. Kameneva, Hemorheology, in: Hemodynamical Flows: Modelling, Analysis and Simulation, G. P. Galdi et al. (eds.), Birkhäuser, 2007, to appear.

[16] A. Sequeira and J. Janela, An overview of some mathematical models in blood rheology, in: A Portrait of Research in the Technical University of Lisbon, M. S. Pereira (ed.), Springer, 2007.

[17] F. N. Van de Vosse et al., Finite-element-based computational methods for cardiovascular fluid-structure interaction, Journal of Engineering Mathematics 47 (2003), 335-368. 
